\title{
Phytosociology of temperate forest at Tinjure-Milke-Jaljale (TMJ) area, Nepal
}

\author{
Bhabindra Niroula* and Shiva Kumar Rai \\ Department of Botany, Post Graduate Campus, Tribhuvan University, Biratnagar, Nepal \\ *E-mail: niroulab@gmail.com
}

Key words: Castanopsis, Quercus, Density, Girth, IVI, Rhododendron

\author{
DOI: http://dx.doi.org/10.3126/on.v16i1.22133 \\ Manuscript details: Received: 12.07.2018 / Accepted: 22.10.2018 \\ Citation: Niroula, B. and S.K. Rai 2018 Phytosociology of temperate forest at Tinjure-Milke-Jaljale (TMJ) area, Nepal, Our Nature \\ 16(1): 80-81. DOI: http://dx.doi.org/ 10.3126/on.v16i1.22133 \\ Copyright: () Niroula and Rai 2018. Creative Commons Attribution - Non Commercial 4.0 International License.
}

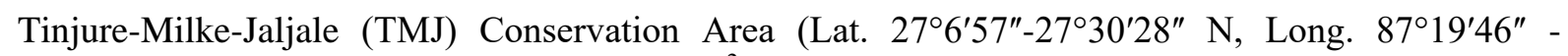
$87^{\circ} 38^{\prime} 14^{\prime \prime}$ E; alt. 1700-5000 m; area $585.26 \mathrm{~km}^{2}$ ) lies between Arun and Tamor rivers in eastern Nepal. This area is popular for Rhododendron, the national flower of Nepal, and has been recognized by "The capital of Rhododendron". Major vegetation types of the area consist of sub-tropical, temperate, subalpine and alpine elements. Mean annual rainfall is $1650 \mathrm{~mm}$, mean temperature $2-22^{\circ} \mathrm{C}$ and characterized by extensive Rhododendron forest, high biodiversity, subsistence livelihood and poverty leading to high dependency on natural resources, deforestation and environmental degradation (IUCN, 2010).

In view of long-term conservation strategy, present report aims to communicate status of selected forests along the Basantapur $(2200-2350 \mathrm{~m})$, Deurali $(2400-2650 \mathrm{~m})$, Tinjure $(2700-2850 \mathrm{~m})$ of Terhathum district and Milke (2850-3050m) of Sankhuwasabha district. Phytosociology of the trees was recorded by quadrate method.

Present study identified a total 14 tree species in TMJ area (Table 1). Pure Rhododendron forest was found in Tinjure and Milke. Density, stump density and total basal cover area of Rhododendron were more in Tinjure forest than in Milke but dead standing, dead fallen and girth $-\mathrm{CBH}$ were more in

Table 1. List of tree species in the sampling areas at Basantapur, Deurali, Tinjure and Milke.

\begin{tabular}{clcc}
\hline SN & Tree species & Local name & Family \\
\hline 1. & Alnus nepalensis D. Don & Utis & Betulace \\
2. & Berberis aristata DC. & Chutro & Berberidaceae \\
3. & Castanopsis tribuloides (Sm.) A. DC. & Katus & Fagaceae \\
4. & Lyonia ovalifolia (Wall.) Drude & Angeri & Ericaceae \\
5. & Mahonia nepaulensis DC. & Keshar & Berberidaceae \\
6. & Persea odoratissima (Nees) Kostem & Kaulo & Lauraceae \\
7. & Pieris formosa (Wall.) D. Don & - & Ericaceae \\
8. & Prunus cerasoides D. Don & Paiyu & Rosaceae \\
9. & Quercus semecarpifolia Sm. & Kharsu & Fagaceae \\
10. & Rhododendron arboreum Sm. & Laligurans & Ericaceae \\
11. & R. thomsonii Hook. f. & Gurans & Ericaceae \\
12. & Rhus javanica L. & Bhalayo & Anacardiaceae \\
13. & Symplocos ramosissima Wall. ex G. Don & Kharane & Symplocaceae \\
14. & S. theifolia D. Don & - & Symplocaceae \\
\hline
\end{tabular}




\section{Our Nature | December $2018 \mid 16$ (1)}

Milke forest than in Tinjure (Table 2). More girth of Rhododendron in Milke was due to mature forest. A few dead standing and absence of dead fallen trees in the Tinjure area indicated more human encroachment. Density, total basal area and IVI of Rhododendron companulatum along the elevation gradient (3600-4100 m) of Manaslu conservation area were $140-940$ tree/ha; $1.0-10.81 \mathrm{~m}^{2} / \mathrm{ha}$ and 138 180, respectively (Rana et al., 2016).

In Deurali forest, Quercus semecarpifolia was dominant and Rhododendron arboreum was codominant trees whereas in Basantapur, Castanopsis tribuloides was dominant and Symplocos ramosisisima was co-dominant trees (Table 3).

Table 2. Status of pure Rhododendron forest at Tinjure and Milke. $(n=10$, mean \pm SE).

\begin{tabular}{lcc}
\hline Parameter & Tinjure area & Milke area \\
\hline Density (tree/ha) & $2870 \pm 16.9$ & $1630 \pm 12.7$ \\
Stump density (stump/ha) & $340 \pm 5.8$ & $330 \pm 5.7$ \\
Dead standing(tree/ha) & $40 \pm 2.0$ & $210 \pm 4.5$ \\
Dead fallen (tree/ha) & 0 & $110 \pm 3.3$ \\
Girth -CBH (cm) & $59.2 \pm 2.4$ & $69.6 \pm 2.6$ \\
Total basal cover area (m²/ha) & $80 \pm 2.8$ & $62.9 \pm 2.5$ \\
\hline
\end{tabular}

Table 3. Girth $(\mathrm{CBH})$, frequency, density, total basal cover area (TBA) and importance value index (IVI) of tree species at Deurali and Basantapur forests. $(n=10$, mean $\pm S E)$

\begin{tabular}{|c|c|c|c|c|c|c|}
\hline $\mathbf{S N}$ & Tree species & $\mathrm{CBH}(\mathbf{c m})$ & Freq. $(\%)$ & $\begin{array}{c}\text { Density } \\
\text { (tree/ha) }\end{array}$ & TBA $\left(\mathbf{m}^{2} / \mathbf{h a}\right)$ & IVI \\
\hline & Deurali area & & & & & \\
\hline 1 & Quercus semecarpfolia & $184.2 \pm 4.2$ & 100 & $580 \pm 7.6$ & $156.4 \pm 3.9$ & $173.1 \pm 4.1$ \\
\hline 2 & Rhododendron arboreum & $93.9 \pm 3.1$ & 80 & $480 \pm 6.9$ & $36.3 \pm 1.8$ & $94.5 \pm 3.1$ \\
\hline 3 & Mahonia nepaulensis & $75.3 \pm 2.7$ & 20 & $50 \pm 2.2$ & $2.0 \pm 0.2$ & $14.0 \pm 1.1$ \\
\hline 4 & Symplocos ramosisisima & $108.1 \pm 3.2$ & 20 & $20 \pm 1.4$ & $1.8 \pm 0.4$ & $11.2 \pm 1.1$ \\
\hline 5 & $\begin{array}{l}\text { Lyonia ovalifolia } \\
\text { Basantapur area }\end{array}$ & $53.3 \pm 2.3$ & 10 & $30 \pm 1.7$ & $0.7 \pm 0.02$ & $7.2 \pm 0.8$ \\
\hline 1 & Castanopsis tribuloides & $57.5 \pm 2.7$ & 100 & $990 \pm 9.9$ & $25.9 \pm 1.6$ & $126.1 \pm 3.5$ \\
\hline 2 & Symplocos ramosisisima & $53.6 \pm 2.3$ & 90 & $410 \pm 6.4$ & $9.34 \pm 3.1$ & $61.0 \pm 1.5$ \\
\hline 3 & Pieris formosa & $34.7 \pm 1.8$ & 50 & $480 \pm 6.9$ & $4.57 \pm 2.1$ & $43.9 \pm 2.1$ \\
\hline 4 & Rhododendron arboreum & $38.8 \pm 1.9$ & 40 & $130 \pm 3.6$ & $1.52 \pm 0.3$ & $19.2 \pm 1.3$ \\
\hline 5 & Symplocos theifolia & $51.5 \pm 2.2$ & 30 & $40 \pm 2.0$ & $0.84 \pm 0.02$ & $10.9 \pm 1.0$ \\
\hline 6 & Prunus cerasoides & $118.5 \pm 3.4$ & 10 & $20 \pm 2.0$ & $2.44 \pm 0.4$ & $8.6 \pm 0.9$ \\
\hline 7 & Unknown (Lauraceae) & $51.5 \pm 2.2$ & 20 & $40 \pm 2.0$ & $0.34 \pm 0.02$ & $7.4 \pm 0.8$ \\
\hline 8 & Berberis aristata & $16.2 \pm 1.2$ & 20 & $30 \pm 1.7$ & 0.06 & $6.3 \pm 0.7$ \\
\hline 9 & Lyonia ovalifolia & $23.6 \pm 1.5$ & 20 & $20 \pm 2.0$ & 0.08 & $5.9 \pm 0.7$ \\
\hline 10 & Alnus nepalensis & $48.3 \pm 2.1$ & 10 & $20 \pm 2.0$ & 0.36 & $4.1 \pm 0.6$ \\
\hline 11 & Mahonia nepaulensis & $36.2 \pm 1.8$ & 10 & $10 \pm 1.0$ & 0.14 & $3.5 \pm 0.5$ \\
\hline 12 & Persea odoratissima & $42.1 \pm 2.0$ & 10 & $10 \pm 1.0$ & 0.10 & $3.1 \pm 0.05$ \\
\hline
\end{tabular}

This is a preliminary work and is recommended for detail socio-economic, taxonomic and ecological studies of TMJ area for long term management and conservation. Authors are grateful to Department of Botany, Post Graduate Campus, T.U., Biratnagar for managing field study.

\section{References}

IUCN 2010. Tinjure-Milke-Jaljale Rhododendron Conservation Area: A strategy for sustainable development. IUCN Nepal Country Office.

Rana, P., M. Koirala, D.R. Bhuju and C. Boonchird 2016. Population structure of Rhododendron companulatum D. Don and associated tree species along the elevational gradient of Manaslu Conservation Area, Nepal. JIST 2(1): 95-102. 